JOURNAL OF POROUS MEDIA

(BEGELL HOUSE PUBLISHING, USA)

$\underline{\text { Impact Factor }=1.035}$

ISSN Print: 1091-028X

ISSN Online: 1934-0508

$\underline{\text { Accepted March } 15^{\text {th }} 2016}$

\title{
STEFAN BLOWING, NAVIER SLIP AND RADIATION EFFECTS ON THERMO-SOLUTAL CONVECTION FROM A SPINNING CONE IN AN ANISOTROPIC POROUS MEDIUM
}

\author{
M.J. Uddin * \\ Mathematics Department, American Int. University-Bangladesh, Dhaka, 1213, Bangladesh. \\ O. Anwar Bég \\ Fluid Mechanics, Aeronautical/ Mechanical Engineering, Computing, Science and Engineering, \\ University of Salford, Manchester, M54WT, England, UK \\ Eemaan T. Bég \\ Engineering Mechanics Associates, Dickenson Road, Manchester, M16, England, UK. \\ * Author for correspondence; Email-jashim_74@yahoo.com
}

\begin{abstract}
Thermal radiation features in many high temperature materials processing operations. To evaluate the influence of radiative flux on spin coating systems, we consider herein the thermo-solutal (coupled heat and mass transfer) in steady laminar boundary layer natural convection flow from a rotating permeable vertical cone to an anisotropic Darcian porous medium. Surface slip effects are also included in the model presented. The conservation equations are rendered into self-similar form and solved as an ordinary differential two-point boundary value problem with surface and free stream boundary conditions using MAPLE 17 software. The transport phenomena are observed to be controlled by ten parameters, viz primary and secondary Darcy numbers ( $D a_{x}$ and $\left.D a_{\theta}\right)$, rotational (spin) parameter $\left(N_{R}\right)$, velocity slip parameter $(a)$, suction/injection parameter $(S)$, thermal slip parameter $(b)$, mass slip parameter $(c)$ buoyancy ratio parameter $(N)$, and conduction-radiation parameter $\left(R_{c}\right)$. Tangential velocity and temperature are observed to be enhanced with greater momentum slip whereas swirl velocity and concentration are reduced. Increasing swirl Darcy number strongly accelerates both the tangential and swirl flow and also heats the regime whereas it decreases concentrations. Conversely a rise in tangential Darcy number accelerates only the tangential flow and decelerates swirl flow, simultaneously depressing temperatures and concentrations. Increasing thermal slip accelerates the swirl flow and boosts concentration but serves to retard the tangential flow and decrease temperatures. With higher radiation contribution (lower $R_{c}$ values) temperatures are elevated and concentrations are reduced. Verification of the MAPLE 17 solutions is achieved using a Keller-box finite difference method $(\boldsymbol{K B M})$. A number of interesting features in the thermo-fluid and species diffusion characteristics are addressed.
\end{abstract}

Key words: Stefan blowing; Spinning cone; MAPLE 17; Anisotropic porous medium; Slip; Keller Box. 


\section{INTRODUCTION}

Heat and mass transfer in the presence of thermal radiation arises in numerous technological applications including paint surface control (Wijewardane and Goswami 2012), combustion chambers (Malico 2001), thermal insulation systems (Kamdem and Baillis 2010), solar cell technologies (Dahan et al. 2013) and materials processing (Viskanta 1999). The complex nature of the integro-differential equation of radiative transfer (Modest 1993) leads to significant difficulty in evaluating thermal radiation distributions even in simple geometric problems. When coupled with convective heat and mass transfer, the conservations equations are even more complex. Engineers have therefore generally adopted the algebraic flux approximations in simulating radiative heat transfer coupled with convective flows. These approximations are of varying sophistication and applicability. They include, in increasing order of complexity, the Rosseland model (Uddin et al. 2014) for gray media, Cogley-Vincenti-Giles approximation (for non-gray gases) (Cogley et al. 1968), Schuster-Schwartzchild two flux approximation (Dembele et al. 2000), P1 approximation (Bogatyreva et al. 2015) and the Chandrasekhar discrete ordinates approximation (Varnas and Truelove 1995). Computational simulation of convective-radiative flows has been an intensive area of scientific endeavor for over two decades, owing to great progress in the efficiency of algorithms and computing power. Yang and Ebadian (1991) studied forced convectionradiation in gray pipe flow, using the method of moments and a SIMPLE algorithm, observing that radiative flux dominates at low Peclet number rather than at a high Peclet number. Dehghan and Behnia (1996) investigated coupled free convection, conduction and radiation heat transfer in an open top upright cavity with a discrete heat source, for variable surface emissivity with a finite element method, noting that radiation generates a recirculation zone within the cavity.

Porous media radiation heat transfer is also of great relevance to combustion systems, contamination in radioactive waste storage in geological systems etc. The general approach for porous media hydrodynamics is the Darcy law which accurately describes pressure drop in a material at low Reynolds numbers (viscous-dominated flows). Takhar et al. (1998) employed the near equilibrium Cogley-Vincenti-Giles radiative flux approximation to analyze boundary layer convection from a vertical plate in porous media with a finite difference code. They observed that heat transfer rates to the wall 
decreased with greater radiation flux whereas temperatures and thermal boundary layer thickness increased. Increasing Darcy number (permeability) was also found to accelerate flows. Tierney et al. (2009) studied methane combustion in porous media using ANSYS software, a modified radiative flux model and the Ergun modified porous drag force model. Cherif and Sifaoui (2004) used a fourth-order finite difference method to simulate radiation effect on heat transfer by simultaneous convection conduction and radiation about a semi-transparent cylindrical porous medium. Moufekkir et al. (2013) employed a combined hybrid lattice Boltzmann, finite difference method and discrete ordinates method to model the coupled heat and mass transfer in a square cavity containing emitting and absorbing gray gas. They observed that stronger volumetric radiation accelerates the flow and modifies the concentration and temperature fields.

The studies alluded above have ignored rotational effects, which are common in a variety of chemical processing systems including surface treatment (Frenot and Chronakis 2003). Such devices may also be modified with porous media for filtration technologies (Holeschovsky and Cooney 1991). These designs exploit the centrifugal force generated by spinning, to modify momentum, heat and mass transfer rates. Many computational analyses of such flows have been reported in the presence of multiple physico/chemical effects. Bég et al. (2008) employed a network electro-thermal code to simulate unsteady Couette flow in a rotating porous medium channel, using a DarcyForchheimer drag force model and evaluating the effects of Ekman number, Forchheimer number, Darcy number and Reynolds number on velocity profiles, carefully studying the sensitivity of flow to these parameters. Kendoush (2013) studied thermal convective heat transfer rates from a rotating, isothermal, porous disk, observing that heat transfer rate is boosted with greater rotational Reynolds number and with a reduction in ordinary Reynolds number. Saxena and Srivastava (2008) investigated viscous flow in a spherical annulus formed by a solid sphere rotating with a constant angular velocity and a concentric spherical porous medium at low Reynolds numbers, employed Brinkman's equation with Ochoa-Tapia and Whitaker interfacial boundary conditions. They observed that the rotational velocity in both the media increases with a rise in permeability and furthermore torque on the rotating sphere is elevated with the reducing permeability. 
Arunachalam et al. (2014) examined rotating hydromagnetic reactive natural convection heat and mass transfer from a vertical sheet in a rotating porous medium. Kumar et al. (2015) studied impact of anisotropy on geometrical and thermal conductivity of metallic foam structures. Al-Harbi (2011) used a finite difference code to analyze unsteady mixed convection flow on a rotating cone embedded in a non-Darcian porous medium saturated with a rotating fluid, computing skin friction, Nusselt number and Sherwood number.

In the processing of certain polymers and industrial fluids, slip effects arise at the interface of the fluid and the boundary. This may exert an important effect on the heat and mass transfer rates at the surface of the body. The presence of slip violates the conventional no-slip boundary condition used in closing boundary layer flow problems. Slip effects can be accommodated in careful modification of wall conditions for temperature (thermal slip), velocity (momentum slip) and concentration (solutal slip). Engineers have therefore studied a variety of slip phenomena in recent years for many different configurations. Lahjomri and Oubarra (2013) developed non-similar and local similarity solutions for slip flow and convection, observing that skin friction coefficient is markedly decreased as the flow becomes more rarefied, and that wall heat transfer is elevated for liquids flow. Oubarra, et al. (2013) considered fully developed slip flow mixed convection in vertical microducts of polygonal and rectangular cross-section, with first-order slip boundary conditions at the walls, noting that Nusselt number and pressure drop are reduced with greater Knudsen number (wall slip). Javed et al. (2009) used a homotopy method to study partial slip effects on axisymmetric stagnation flow with heat transfer. Yu and Ameel (2001) considered thermal slip effects in microchannels. Bég et al. (2015) studied multiple slip effects on unsteady magnetohydrodynamic nanofluid transport with heat generation/absorption effects in temperature dependent porous media

In certain manufacturing processes (and indeed in all geological materials), anisotropy is encountered. This relates to the variation of permeability in different directions. The over-whelming majority of mathematical models for transport phenomena in porous media have employed an isotropic Darcian formulation i.e. they assume that permeability is the same in any direction. Anisotropic heat and mass transfer studies have however received some limited attention. Relevant investigations in this regard include Dhanasekaran et al. (2001) who analyzed cylindrical cavities containing anisotropic 
porous materials. Kumar and Bera (2009) investigated numerically with a spectral element method, the free convection in a hydrodynamically anisotropic porous enclosure using a Brinkman-extended non-Darcy model and implementing a permeability tensor with principal direction oblique to the gravity vector. They found that contrary to isotropic porous media, enhancement of thermal convection in the anisotropic porous enclosure does not require elevation in the lateral heat transfer rate, and that anisotropic permeability strongly modifies base and side average Nusselt numbers. Further studies of anisotropic porous media convection include Alex and Patil (2001).

All of the previous researchers ignored the normal interfacial velocity due to species (mass) diffusion. However, there are many real life situations (e.g. in paper drying process) where species (mass) transfer happens by evaporation. Based on the temperature and the water content of the wet paper sheet, species transfer may be significant and may produce a "blowing effect'. This blowing effect initiates from the idea of the Stefan problem related to species transfer (Nellis and Klein 2008). Species diffusion produces a bulk motion of fluid which generates additional motion of the fluid. This has been described in text books on heat and mass transfer (Lienhard and Lienhard 2005) and in an excellent monograph concerning heat and mass transfer in magnetohydrodynamic (MHD) flows (Blums et al. 1987). To reflect the blowing effects for large mass transfer flux, blowing parameter is used to provide a correction factor when using traditional results without using blowing effects. This blowing effect is different from the mass injection or blowing because of transpiration where the surface is considered as permeable. Nonetheless for the blowing effects used in this paper, it is for impermeable surface not permeable surface and the blowing is because of transfer flux of certain species from the surface (ambient) to the ambient (surface). Species transfer depends on the flow field, and the flow field is influenced by the mass blowing at the wall. All of these generate a coupling between momentum and concentration fields. The blowing effects have been investigated in the literature for different flow configurations. Fang and 
Jing (2014) investigated the flow, heat and mass transfer of a viscous fluid flow past a stretching sheet by considering the blowing effects of mass transfer under high flux conditions. Fang (2014) studied the flow and mass transfer of a two-dimensional unsteady stagnation-point flow over a moving wall, considering the coupled blowing effect from mass transfer.

It is now known that anisotropic porous media models provide a more representative description of directional variation in the permeability encountered in many industrial materials, filters, soils etc. In the present study we consider theoretically the combined convective heat and mass transfer from a rotating cone to anisotropic Darcian porous media with multiple walls slip effects and radiative flux. A MAPLE numerical solution (Uddin et al. 2014), based on Runge-Kutta-Fehlberg shooting algorithms, is developed for the transformed nonlinear boundary value problem. The computations are verified with the implicit second order accurate Keller box finite difference method (Keller 1976). A detailed parametric examination of emerging thermophysical parameters on the momentum, heat and mass transfer (species diffusion) characteristics is conducted. To the authors' knowledge this is the first analytical and numerical study of its kind reported in the literature.

\section{DOUBLE-DIFFUSIVE RADIATIVE CONVECTION SLIP MODEL}

Axisymmetric, steady-state, laminar, incompressible, natural convection-radiative thermo-solutal boundary layer flow is examined, along a rotating cone embedded in a high permeability, anisotropic saturated porous regime. The geometry of the problem is illustrated in Figure 1.

Buoyancy forces are generated by both temperature difference and concentration difference. Tortuosity, matrix deformability and thermal dispersion effects in the porous medium are neglected. The Darcy model is employed (Bég and Makinde 2011). The fluid is optically thick. Rotation is sufficiently slow to ignored fluid compressibility effects. The cone surface temperature and concentration vary linearly with $X$ (the cone surface is non-isothermal and non-isosolutal). The $X$-direction is parallel to the cone slant surface, the $Y$ - direction normal to this and $\theta$ - designates the angle in a plane 
perpendicular to the vertical symmetry axis. The cone is a representative geometry for chemical engineering mixing devices. The governing equations for the flow regime may be presented, with reference to an $(X, Y, \theta)$ coordinate system, as follows:

\section{Mass:}

$$
\frac{\partial(R U)}{\partial X}+\frac{\partial(R V)}{\partial Y}=0
$$

\section{Tangential Momentum}

$$
U \frac{\partial U}{\partial X}+V \frac{\partial U}{\partial Y}-\frac{W^{2}}{R} \frac{d R}{d X}=v \frac{\partial^{2} U}{\partial Y^{2}}-\frac{v}{K_{X}} U+g \beta_{T} \cos \gamma\left(T-T_{\infty}\right)+g \beta_{C} \cos \gamma\left(C-C_{\infty}\right),
$$

\section{Swirl Momentum}

$$
U \frac{\partial W}{\partial X}+V \frac{\partial W}{\partial Y}+\frac{U W}{R} \frac{d R}{d X}=v \frac{\partial^{2} W}{\partial Y^{2}}-\frac{v}{K_{\theta}} W
$$

\section{Energy:}

$U \frac{\partial T}{\partial X}+V \frac{\partial T}{\partial Y}=\alpha \frac{\partial^{2} T}{\partial Y^{2}}-\frac{1}{\rho c_{p}} \frac{\partial q_{r}}{\partial Y}$

\section{Species (Concentration):}

$$
U \frac{\partial C}{\partial X}+V \frac{\partial C}{\partial Y}=D \frac{\partial^{2} C}{\partial Y^{2}}
$$

The Boussinesq approximation has been used so that buoyancy effects only appear in the $X$-direction momentum Eqn. (2), which is coupled to the energy and concentration equation, constituting a free convection regime. Using separate permeabilities in the $X$ and $\theta$ directions (due to anisotropy), two porous media drag force terms are present, one in each of Eqns. (2) and (3) i.e. the primary and secondary Darcian impedance. The corresponding boundary conditions at the surface and far from the cone are, following Fang (2014):

$$
\begin{aligned}
& U=N_{1} v \frac{\partial U}{\partial Y}, V=-\frac{D}{(X / L)\left(1-C_{w}\right)}\left(\frac{\partial C}{\partial Y}\right), W=\Omega R+N_{2} v \frac{\partial W}{\partial Y}, \\
& T=T_{\infty}+\left(T_{w}-T_{\infty}\right)(X / L)+D_{1} \frac{\partial T}{\partial Y}, C=C_{\infty}+\left(C_{w}-C_{\infty}\right)(X / L)+E_{1} \frac{\partial C}{\partial Y} \text { at } Y=0, \\
& U=W=0, T=T_{\infty}, C=C_{\infty} \text { as } Y \rightarrow \infty .
\end{aligned}
$$


where the following notation applies: $X$-coordinate parallel to cone surface, $Y$ coordinate normal to cone surface, $\theta$ - angular coordinate, $R$-radial coordinate, $r$-local radius of the cone, $U$ - velocity component in $X$ direction, $V$-velocity component in $Y$ direction, $W$ - velocity component in $\theta$ direction, $T$ - fluid temperature, $T_{w}$ - cone surface temperature, $T_{\infty}$-free stream temperature, $C$ - fluid concentration, $C_{w}$ - cone surface concentration, $C_{\infty}$ - free stream concentration, $g$ - gravitational acceleration, $v$ kinematic viscosity of fluid, $\rho$ - density of fluid, $K_{X}$ - permeability in $X$ direction , $K_{\theta}$ permeability in $\theta$ direction, $\alpha$ - thermal diffusivity of the fluid, $D$ - mass diffusivity of the fluid, $\beta_{T}$ - coefficient of thermal expansion of the fluid, $\beta_{C}$-coefficient of mass expansion of the fluid $\Omega$ - rotational velocity of the cone (spin velocity about symmetry axis), $\gamma$ semi-vertex angle of cone, $q_{r}=-\frac{4 \sigma_{1}}{3 k_{1}} \frac{\partial T^{4}}{\partial Y}-$ radiative heat flux, $\sigma_{1}\left(=5.67 \times 10^{-8} \mathrm{~W} / \mathrm{m}^{2}\right.$ $\left.\mathrm{K}^{4}\right)$-Stefan-Boltzmann constant, $k_{1}\left(\mathrm{~m}^{-1}\right)$-Rosseland mean absorption coefficient, $N_{1}$ velocity slip factor for the $u$-velocity component, $N_{2}$-velocity slip factor for the $W$ velocity component, $D_{1}$ - thermal slip factor, $E_{1}$ - mass slip factor. For uniform roughness $N_{1}=N_{2}$ (e.g. spinning disk used in reaction vessels) whilst for concentric grooves (e.g. phonograph record, laser-etched disk, hard disk platters, spiral grooved disks used in fluid separators etc.) $N_{1}<N_{2}$, for radial grooves (e.g. brake disks, Nipkow spinning disks used in spinning disk confocal microscopy, radial grooved disks used in fluid mixers) $N_{1}>N_{2}$. For the sake of simplicity, in this study we assumed that $N_{1}=N_{2}$. The boundary layer Eqns. (1) to (5) is highly coupled, parabolic and nonlinear. An analytical solution is clearly intractable and in order to obtain a robust solution, we next non-dimensionalize the model. Proceeding with the analysis, we introduce the following transformations:

$$
\begin{aligned}
& x=\frac{X}{L}, y=\frac{Y}{L G r_{L}^{-1 / 4}}, r=\frac{R}{L}, u=\frac{U}{U^{*}}, v=\frac{V}{U^{*} G r_{L}^{-1 / 4}}, w=\frac{W}{\Omega L}, D a_{x}=\frac{K_{X} U^{*}}{v L}, \\
& D a_{\theta}=\frac{K_{\theta} U^{*}}{v L}, S c=\frac{v}{D}, U^{*}=\left[g \cos \gamma \beta_{T} L\left(T_{w}-T_{\infty}\right)\right]^{1 / 2}, \Theta=\frac{T-T_{\infty}}{T_{w}-T_{\infty}}, \\
& \Phi=\frac{C-C_{\infty}}{C_{w}-C_{\infty}}, G r=\left[\frac{U * L}{v}\right]^{2}, \operatorname{Re}=\frac{\mathrm{W} L^{2}}{n}, a=\frac{N_{1} v}{L G r^{-1 / 4}}, b=\frac{D_{1}}{L G r^{-1 / 4}} \\
& S=\frac{\left(C_{w}-C_{\infty}\right) v}{\left(1-C_{w}\right) U^{*} L G r^{-1 / 2}}, c=\frac{E_{1}}{L G r^{-1 / 4}}, N=\frac{\beta_{C}\left(C_{w}-C_{\infty}\right)}{\beta_{T}\left(T_{w}-T_{\infty}\right)}, R_{c}=\frac{k k_{1}}{4 \sigma_{1} T_{\infty}^{3}}, \operatorname{Pr}=\frac{v}{\alpha} .
\end{aligned}
$$


$U^{*}$ - reference velocity. The transport equations are thereby reduced to the following dimensionless equations:

Mass:

$\frac{\partial(r u)}{\partial x}+\frac{\partial(r v)}{\partial y}=0$

Tangential Momentum

$u \frac{\partial u}{\partial x}+v \frac{\partial u}{\partial y}-\frac{\operatorname{Re}^{2}}{G r} \frac{w^{2}}{r}=\frac{\partial^{2} u}{\partial y^{2}}-\frac{u}{D a_{x}}+\Theta+N \Phi$,

\section{Swirl Momentum}

$u \frac{\partial w}{\partial x}+v \frac{\partial w}{\partial y}+\frac{u w}{r}=\frac{\partial^{2} w}{\partial y^{2}}-\frac{w}{D a_{\theta}}$,

\section{Thermal Energy (Heat):}

$u \frac{\partial \Theta}{\partial x}+v \frac{\partial \Theta}{\partial y}=\frac{1}{\operatorname{Pr}}\left(1+\frac{4}{3 R_{c}}\right) \frac{\partial^{2} \Theta}{\partial y^{2}}$,

\section{Species:}

$u \frac{\partial \Phi}{\partial x}+v \frac{\partial \Phi}{\partial y}=\frac{1}{S c} \frac{\partial^{2} \Phi}{\partial y^{2}}$

The boundary conditions are also transformed to:

$$
\begin{aligned}
& u=a \frac{\partial u}{\partial y}, v=-\frac{D\left(C_{w}-C_{\infty}\right)}{2 x\left(1-C_{w}\right) U^{*} L G r^{-1 / 2}} \frac{\partial \Phi}{\partial y}, w(x, 0)=r+a \frac{\partial w}{\partial y}, \\
& \Theta=x+b \frac{\partial \Theta}{\partial y}, \Phi=x+c \frac{\partial \Phi}{\partial y} \text { at } y=0 \\
& u=w=\Theta=\Phi=0 \text { as } y \rightarrow \infty .
\end{aligned}
$$

In Eqns. (9)-(15), the following notation applies: $x$-transformed $X$ coordinate, $y$ transformed $Y$ coordinate, $r$ - transformed local cone radius, $u$-transformed $X$ velocity, $v$ - transformed $Y$ velocity, $w$ - transformed $\theta$ - velocity, Da $a_{x}$ tangential Darcy number, $D a_{\theta}$ - (swirl Darcy number), Pr - Prandtl number, Sc - Schmidt number, $\Theta$ - non- 
dimensional temperature function, $\Phi$ - non-dimensional concentration function, $G r$ Grashof number, Re - rotational Reynolds number, L - reference scale length, $N$ buoyancy ratio parameter, $R_{c}$-radiation-conduction parameter, a-momentum slip parameter, $b$ - thermal slip parameter, $c$ - mass slip parameter.

The non-dimensional Eqns. (9) to (15) can be further simplified by employing appropriate similarity transformations. We first define a dimensional stream function, $\psi$, following Ece (2006):

$$
r u=\frac{\partial \psi}{\partial y}, r v u=-\frac{\partial \psi}{\partial x}
$$

The boundary layer variables are now re-scaled as follows, with $r=x \sin \phi$ :

$\psi(x, y)=x r F(y), w=r G(y), \Theta=x H(y), \Phi=x I(y)$.

Introducing these relations into Eqns. (9)-(15), generates the following $9^{\text {th }}$ order system of "self-similar" ordinary differential equations:

\section{Tangential Momentum:}

$F^{\prime \prime \prime}+2 F F^{\prime \prime}-\left(F^{\prime}\right)^{2}-\frac{F^{\prime}}{D a_{x}}+N_{R} G^{2}+H+N I=0$

\section{Swirl Momentum:}

$G^{\prime \prime}+2 F G^{\prime}-2 F^{\prime} G-\frac{G}{D a_{\theta}}=0$

\section{Energy:}

$\frac{1}{\operatorname{Pr}}\left(1+\frac{4}{3 R_{c}}\right) H^{\prime \prime}+2 F H^{\prime}-F^{\prime} H=0$,

Mass:

$\frac{1}{S c} I^{\prime \prime}+2 F I^{\prime}-F^{\prime} I=0$

The transformed boundary conditions for the current problem take the form: 
$F=\frac{S}{2 S c} I^{\prime}, F^{\prime}=a F^{\prime \prime}, G=1+a G^{\prime}, H=1+b H^{\prime}, I=1+c I^{\prime}$ at the cone surface $(y=0)$ $F^{\prime} \rightarrow 0, G \rightarrow 0, H \rightarrow 0, I \rightarrow 0$ in the freestream $(y \rightarrow \infty)$,

where $F$ is the boundary-layer stream function, $G$ is the boundary-layer rotational (swirl) velocity, $H$ is the boundary-layer temperature, $I$ is the boundary-layer concentration, and $N_{R}=\frac{(\operatorname{Re} \sin \gamma)^{2}}{G r}$ is the rotational (spin) parameter, $S$ is the suction/injection parameter, $N$ is the buoyancy ratio parameter, $R_{c}$ is the convection-radiation parameter. Note that $S>0$ for species transfer from the wall to the free stream (i.e., evaporation) whilst $S<0$ for species transfer from the free stream to the wall (i.e., condensation). In other words, for $S$ $>0$, there exists mass blowing at the wall and for $S<0$ it becomes mass suction.

We further note that for the purely fluid case, $D a_{x} \rightarrow \infty$ and $D a_{\theta} \rightarrow \infty$, since the permeability of the anisotropic regime becomes infinite. For the case of an isotropic porous medium, $D a_{x}=D a_{\theta}$. and in the absence of mass equations, in the absence of radiation $\left(R_{c} \rightarrow \infty\right)$, the self-similar momentum equations (18) and (19) and energy equation (20) then reduce to exactly the non-magnetic case of the generalized hydromagnetic equations and energy equation solved by Ece (2006):

$F^{\prime \prime \prime}+2 F F^{\prime \prime}-\left(F^{\prime}\right)^{2}+N_{R} G^{2}+H=0$,

$G \notin+2 F G \notin-2 F \notin=0$.

$\frac{1}{\operatorname{Pr}} H^{\prime \prime}+2 F H^{\prime}-F^{\prime} H=0$,

In the absence of slips and blowing parameters, the transformed boundary conditions are also identical to those solved by Ece (2006) and for the current problem take the form: $F=0, F^{\prime}=0 ; G=1, H=1$ at the cone surface $(y=0)$, $F^{\prime} \rightarrow 0, G \rightarrow 0, H \rightarrow 0$ in the freestream $(y \rightarrow \infty)$.

\section{NUMERICAL SOLUTION BY MAPLE 17}

The self-similar nonlinear two-point boundary value problem is solved using MAPLE17 (Uddin et al. 2013). This approach has been extensively implemented in recent years. 
Applications include nanofluid flow in porous media (Uddin et al. 2012), magnetic heat transfer in rotating porous media (Bég et al. 2012) and thermo-solutal slip flow (Bég et al. 2014). A Runge-Kutta-Fehlberg fourth-fifth order numerical algorithm (RKF45) is employed, available in the symbolic computer software Maple 17. This utilizes a collocation method in which a finite-dimensional space of candidate solutions is selected (usually, polynomials up to a certain degree) and a number of points in the domain (called collocation points), and a solution selected which satisfies the given equation at the collocation points. The RFK45 algorithm is adaptive since it adjusts the quantity and location of grid points during iteration and thereby constrains the local error within acceptable specified bounds. In the current problem, the asymptotic boundary conditions given in Eq. (22) are replaced by a finite value 15. The location of the far boundary must be selected judiciously to ensure that all numerical solutions approached to the asymptotic values correctly. The selection of a sufficiently large domain is imperative for maintaining desired accuracy in boundary layer flows, and is a common pitfall encountered in numerous studies. The stepping formulae used to solve Eqns. (18)(21) under conditions (22) via fifth-fourth order Runge-Kutta-Fehlberg algorithms are given below (Uddin et al. 2013):

$$
\begin{aligned}
& k_{0}=f\left(x_{i}, y_{i}\right) \\
& k_{1}=f\left(x_{i}+\frac{h}{4}, y_{i}+\frac{h}{4} k_{0}\right) \\
& k_{2}=f\left(x_{i}+\frac{3 h}{8}, y_{i}+\left(\frac{3}{32} k_{0}+\frac{9}{32} k_{1}\right) h\right) \\
& k_{3}=f\left(x_{i}+\frac{12 h}{13}, y_{i}+\left(\frac{1932}{2197} k_{0}-\frac{7200}{2197} k_{1}+\frac{7296}{2197} k_{2}\right) h\right) \\
& k_{4}=f\left(x_{i}+h, y_{i}+\left(\frac{439}{216} k_{0}-8 k_{1}+\frac{3860}{513} k_{2}-\frac{845}{41047} k_{3}\right) h\right) \\
& k_{5}=f\left(x_{i}+\frac{h}{2}, y_{i}+\left(-\frac{8}{27} k_{0}+2 k_{1}-\frac{3544}{2565} k_{2}+\frac{1859}{4104} k_{3}-\frac{11}{40} k_{4}\right) h\right) \\
& y_{i+1}=y_{i}+\left(\frac{25}{216} k_{0}+\frac{1408}{2565} k_{2}+\frac{2197}{4104} k_{3}-\frac{1}{5} k_{4}\right) h \\
& z_{i+1}=z_{i}+\left(\frac{16}{135} k_{0}+\frac{6656}{12825} k_{2}+\frac{28561}{56430} k_{3}-\frac{9}{50} k_{4}+\frac{2}{55} k_{5}\right) h
\end{aligned}
$$


Here $y$ denotes fourth-order Runge-Kutta phase and $z$ is the fifth-order Runge-Kutta phase. An estimate of the error is achieved by subtracting the two values obtained. If the error exceeds a specified threshold, the results can be recalculated using a smaller step size. The approach to estimating the new step size is shown below:

$h_{\text {new }}=h_{\text {old }}\left(\frac{\varepsilon h_{\text {old }}}{2\left|z_{i+1}-y_{i+1}\right|_{5}}\right)^{1 / 4}$

\section{KELLER BOX METHOD VERIFICATION COMPUTATIONS}

The MAPLE numerical solutions have been validated using an implicit finite difference scheme, which despite recent developments in other numerical methods, remains a powerful and very accurate approach for boundary layer flow equation systems which are generally parabolic in nature. It is unconditionally stable and achieves exceptional accuracy. An excellent summary of this technique is given in Keller (1976). The method has been used in rotating fluid dynamics (Bég et al. 2014), mixed convection from curved bodies (cylinders) (Bég et al. 2012) and transport in porous media (Javed et al. 2011). Keller's box method (KBM) discretization is fully coupled at each step which reflects the physics of parabolic systems - which are also fully coupled. Discrete calculus associated with the Keller-Box scheme has also been shown to be fundamentally different from all other mimetic (physics capturing) numerical methods, as elaborated in Bég (Prasad et al. 2012). KBM comprises four stages.

1) Decomposition of the $N^{\text {th }}$ order partial differential equation system to $N$ first order equations.

2) Finite Difference Discretization.

3) Quasilinearization of Non-Linear Keller Algebraic Equations and finally.

4) Block-tridiagonal Elimination solution of the Linearized Keller Algebraic Equations

The algebraic details for the present problem are omitted for brevity. In order to prove the convergence and stability of the KBM, the Matlab program ROTAKELLSIM (Rotational Keller Simulation) is executed with slightly modified values of the mesh distance in the $y$-direction i.e. $\boldsymbol{j}$, and no significant change is observed in the values of the velocity components. Mesh independence of solutions is therefore achieved with excellent stability and convergence Prasad et al. 2012). The boundary conditions (22) are easily specified in ROTAKELLSIM. The discretization yields a large linearized algebraic 
system which is solved by the block-elimination method since it possesses a block-tri-diagonal structure (i.e. comprises block matrices). The complete linearized system is formulated as a block matrix system, where each element in the coefficient matrix is a matrix itself, and this system is solved using the efficient Keller-box method. The numerical results are strongly influenced by the number of mesh points in both directions. After some trials in the $\eta$-direction (transverse dimensionless coordinate) a larger number of mesh points are selected. $\eta_{\max }$ has been set at 15 and this defines an adequately large value at which the prescribed boundary conditions are satisfied. Mesh independence is achieved in the present computations i.e. by increasing mesh density no further tangible improvement in solution accuracy is achieved. The numerical algorithm is executed in MATLAB on a PC.

\begin{tabular}{|c|c|c|c|c|c|c|}
\hline \multirow[t]{3}{*}{$N_{R}$} & \multicolumn{6}{|c|}{$F^{\prime \prime}(0)$} \\
\hline & \multicolumn{3}{|l|}{$\operatorname{Pr}=1$} & \multicolumn{3}{|l|}{$\operatorname{Pr}=10$} \\
\hline & $\begin{array}{l}\text { Ece } \\
(2006)\end{array}$ & KBM & Maple17 & $\begin{array}{l}\text { Ece } \\
(2006)\end{array}$ & KBM & Maple17 \\
\hline 0 & 0.68150212 & 0.6814929 & 0.6814833 & 0.43327726 & 0.4321874 & 0.4291876 \\
\hline 0.5 & 0.84650616 & 0.8464987 & 0.8464882 & 0.62601869 & 0.6230851 & 0.6228014 \\
\hline 1.0 & 1.00196008 & 1.0020111 & 1.0019431 & 0.79828572 & 0.7983791 & 0.798418104 \\
\hline 2.0 & 1.29230021 & 1.2924014 & 1.2922849 & 1.10990481 & 1.1098976 & 1.10990496 \\
\hline
\end{tabular}

Table 1: Values of $\mathrm{F}^{\prime \prime}(0)$ for free-convection boundary-layer flow over a spinning cone with $D a_{x} \rightarrow \infty$ and $D a_{\theta} \rightarrow \infty$ (purely fluid case), $a=b=c=0, R_{c} \rightarrow \infty$, neglecting species eqn. (21).

\begin{tabular}{|c|c|c|c|c|c|c|}
\hline \multirow[t]{3}{*}{$N_{R}$} & \multicolumn{6}{|c|}{$-H^{\prime}(0)$} \\
\hline & \multicolumn{3}{|l|}{$\operatorname{Pr}=1$} & \multicolumn{3}{|l|}{$\operatorname{Pr}=10$} \\
\hline & $\begin{array}{l}\text { Ece } \\
(2006)\end{array}$ & KBM & Maple17 & $\begin{array}{l}\text { Ece } \\
(2006)\end{array}$ & KBM & Maple17 \\
\hline 0 & 0.63886614 & 0.63886429 & 0.63885470 & 1.27552680 & 1.27552692 & 1.26598645 \\
\hline 0.5 & 0.67194897 & 0.67194871 & 0.67193844 & 1.47165986 & 1.47165994 & 1.547638332 \\
\hline 1.0 & 0.70053401 & 0.70053387 & 0.70052453 & 1.60768499 & 1.60768489 & 1.20756132 \\
\hline 2.0 & 0.74869559 & 0.74869472 & 0.74868824 & 1.80575019 & 1.80575025 & 1.80574943 \\
\hline
\end{tabular}


Table 2: Values of $-H^{\prime}(0)$ for free- $\quad$ convection boundary-layer flow over a spinning cone with $D a_{x} \rightarrow \infty$ and $D a_{\theta} \rightarrow \infty$ (purely fluid case), $a=b=c=0, R_{c} \rightarrow \infty$, neglecting species eqn. (21).

Both MAPLE and ROTAKELLSIM (KBM) correlate very closely with the infinite permeability solutions of Ece (2006). Confidence in the MAPLE code is therefore high, and this is deployed to present all graphs.

\section{MAPLE COMPUTATIONS AND DISCUSSION}

Rigorous computations are presented in this section for distributions of non-dimensional tangential velocity $\left(F^{\prime}\right)$, swirl velocity $(G)$, temperature $(H)$ and concentration $(I)$ with distance, $y$, into the boundary layer (transverse to the cone surface). Although 11 thermophysical parameters are featured in the model, we examine explicitly the effects of only nine, viz D $a_{x}$-tangential Darcy number, Da $a_{\theta}$-swirl Darcy number, $N_{R}$ - rotational (spin) parameter (a function of rotational Reynolds number), a - momentum (velocity slip) parameter, parameter, b - thermal slip (jump) parameter, c - mass (solutal) slip parameter, $S$ - wall mass flux (transpiration) parameter ( $>0$ for suction, $<0$ for injection and $=0$ for solid cone), $N$ - buoyancy ratio parameter and $R_{c}$ - conduction-radiation parameter. Both Sc-Schmidt number and Pr-Prandtl number are fixed in the computations and correspond to hydrogen diffusing in air (as encountered in chromotography applications). The porous medium possesses very high permeability in both the $x$ - and $\theta$-directions so that high values are prescribed for $D a_{x}$ and $D a_{\theta}$. i.e. 1.0, unless otherwise stated (the medium is sparsely packed). $S c$ is prescribed as $0.22, \operatorname{Pr}$ is 0.72. $N_{R}=1.0$ (Coriolis force and buoyancy force effects are equivalent) unless otherwise indicated. Furthermore the default value of $R_{c}$ is 1 such that both thermal conduction and thermal radiation contribute equally. All computations are illustrated in Figs. 2-15.

The far boundary is prescribed at $\eta=15$ to ensure asymptotically smooth solutions are attained in the free stream.

In Figs 2-3, the effects of surface transpiration (associated with the perforated cone) on tangential and swirl velocities and temperature and concentration, respectively, are presented. Significant deviations in profiles are observed in the velocity profiles (fig. 2). Tangential profiles are parabolic- they ascend from the cone surface, attain a maximum a short distance therefrom and then descend smoothly to vanish in the free stream. Swirl profiles however follow a consistent monotonic decay from the cone surface to the 
freestream. With greater suction $(S<0)$ both velocity fields are suppressed; with greater injection $(S>0)$ they are both accelerated. The case of the solid cone (no transpiration) naturally falls between the suction and injection (blowing) cases. Suction clearly causes adhesion of the boundary layer to the cone surface and this at sufficiently high rotational Reynolds numbers will delay boundary layer separation. Injection adds momentum to the boundary layer regime and encourages greater viscous diffusion, of interest in certain coating operations. Further from the wall, significantly greater tangential velocity magnitudes are computed compared with swirl velocity magnitudes, even though the latter are greater in the near-wall zone. Fig. 3 demonstrates that both temperature and concentration decay from maxima at the wall (cone surface) to the free stream, irrespective of the transpiration effect. Greater suction however depresses both temperatures and concentration values, although larger magnitudes of concentration are apparent over the entire region transverse to the cone surface. Greater injection values are found to assist thermal and species diffusion, thereby enhancing thermal and concentration boundary layer thicknesses. The destruction of momentum associated with larger wall suction is principally responsible for the reduction in heat and mass diffusion, with the opposite effect sustained for injection.

Figs. 4, 5 illustrate the influence of the momentum (velocity) slip effect $a$ on flow characteristics. Tangential flow is observed to experience a strong acceleration with greater slip, whereas swirl flow is damped (fig. 4). As momentum slip increases, the tangential near-wall peak is progressively displaced closer to the cone surface. The slip parameter, $a$, arises in both velocity boundary conditions at the wall (eqn.22). However since the tangential flow is dominant (i.e. the primary flow) it draws the greater part of momentum from the swirl field and thereby depletes the latter. The boost in tangential flow is therefore primarily aided with greater momentum slip whereas the swirl flow is further retarded. With increasing momentum slip, temperature is weakly enhanced, whereas the species concentration is weakly decreased (fig. 5). In any scenario, concentration magnitudes are however in excess of temperatures i.e. concentration boundary layer thickness exceeds the thermal boundary layer thickness.

Figs. 6,7 present the effect of thermal slip (jump), $b$, on the velocity functions, temperature and concentration function distributions. Thermal slip parameter features only in the augmented wall temperature condition in eqn. (22). It will therefore have a 
pronounced effect on temperatures, but will indirectly (via coupling of all the boundary layer equations to each other), impart an effect on velocity and concentration fields. In fig. 6 we observe that this is indeed the case- tangential flow is seen to be strongly suppressed, with the peak again migrating for stronger thermal slip closer to the cone surface. Swirl flow is however weakly elevated. Fig. 7 reveals that whereas the concentration is enhanced somewhat throughout the boundary layer transverse to the wall, temperatures are very substantially decreased, in particular at the cone surface. Cooling of the cone surface is therefore achieved successfully with the thermal slip effect.

Figs. 8 depicts the response of the species concentration to solutal (mass) slip parameter, c. As anticipated this parameter, solely arising in the modified wall condition for concentration in eqn. (22) does not influence tangential and swirl velocity or temperatures (graphs are therefore omitted), despite the coupling of the concentration field to the tangential field, via the terms $2 F I^{\prime}$ and $-F^{\prime} I$ in eqn. (21). With greater mass slip, there is a marked depression in concentration magnitudes, especially at the wall (cone surface) and this effect is transferred into the boundary layer, although it weakens progressively with further distance from the wall. Evidently therefore species diffusion in the boundary layer is non-trivially decreased with greater mass slip and this will manifest in a thinning in species boundary layer thickness.

Figs. 9 illustrate the influence of the conduction-radiation parameter i.e. $R_{c}=\frac{k k_{1}}{4 \sigma_{1} T_{\infty}^{3}}$ on temperature and concentration distributions. This parameter embodies the relative contribution of thermal conduction and thermal radiative heat transfer. It is a valid approach for optically thick media wherein optical thickness is high and is sometimes known as the Rosseland-Boltzmann parameter. This parameter features as a denominator in the energy eqn. (20), viz $\left(4 / 3 R_{c}\right) H^{\prime \prime}$. As $R_{c}$ increases in value, the radiative contribution is decreased and conductive heat transfer contribution is increased. For $R_{c}=1$ both modes of heat transfer contribute equally. As $R_{c} \rightarrow \infty$, the radiative contribution vanishes and thermal conduction dominates, and for this scenario eqn. (20) contracts to the conventional convection heat transfer equation. Fig. 9 shows that a significant decrease in temperature is induced with greater $R_{c}$ values. The temperature is maximized for the lowest $R_{c}$ value of 1 i.e. the strongest thermal radiation case and minimized for the largest 
value of $R_{c}=5$ i.e. the weakest thermal radiation case. Greater thermal radiation flux therefore exerts the correct effect on temperature distributions i.e. heats the medium. Conversely with weaker thermal radiation (lower $R_{c}$ values), the concentration magnitudes are found to be depressed. Thermal boundary layer thickness is therefore enhanced with greater radiative effect whereas species boundary layer thickness is reduced.

Figs. 10, 11 present the influence of buoyancy ratio $(N)$ on tangential velocity $\left(F^{\prime}\right)$ and swirl velocity $(G)$, and temperature $(H)$ and concentration $(I)$. The buoyancy ratio, $N=\frac{\beta_{C}\left(C_{w}-C_{\infty}\right)}{\beta_{T}\left(T_{w}-T_{\infty}\right)}$ appears in the coupling term, $N I$ in the tangential flow equation (18). It represents the relative influence of species buoyancy force compared with thermal buoyancy force and is a critical parameter in double-diffusive (thermo-solutal) convection flows. When $N=0$ purely forced convection heat and mass transfer arises. For $N>1$ species buoyancy dominates thermal buoyancy and vice versa for $N<1$. Greater $N$ values are found to greatly accentuate the tangential flow but to weakly retard the swirl flow (fig. 10). Both temperature and concentration are depressed with greater buoyancy ratio (fig. 11) although concentration magnitudes are always greater than temperatures. Evidently with greater buoyancy ratio, both thermal and species boundary layer thickness are reduced.

Figs. 12-13 present the evolution of the flow, thermal and species characteristics with swirl Darcy number, $D a_{\theta}$ i.e. $\theta$ - direction permeability function. With greater $D a_{\theta}$ (and these values are intentionally large owing to the sparsely packed nature of the porous medium considered), the tangential velocity, $F^{\prime}$, is noticeably elevated near the cone surface (figure 12), attaining a peak and thereafter plummeting to the vanishing free stream value. This behavior is sustained through the boundary layer for all values of transverse coordinate with all profiles converging in the free stream. The tangential flow is therefore accelerated strongly with greater $D a_{\theta}$ values. In the swirl momentum equation, the inverse proportionality of the Darcian bulk impedance to tangential Darcy number is evident in the drag force term, $-\frac{G}{D a_{\theta}}$, which has the opposite effect to the Coriolis body force. The coupling of the swirl and tangential momenta equations manifests in a strong influence of this Darcian drag impedance on the tangential flow. As 
tangential permeability increases, the porous medium comprises progressively less solid particles (which cause resistance) and becomes increasingly fluid. In chemical filtration processes, greater velocity control is therefore achieved with lower permeabilities in the tangential direction. The porous medium may be designed therefore to capitalize on this effect. Increasing injection at the cone surface physically accelerates the tangential flow whereas increasing suction suppresses it. Swirl velocity, $G$, (figure 12) although also enhanced with greater tangential Darcy number, is less dramatically altered than tangential velocity. The swirl velocity field is also damped out much faster than the tangential field-profiles decay sharply to the free stream value for any value of rotation parameter even the case of a stationary cone $\left(N_{R}=0\right)$. Irrespective of the value of $D a_{\theta}$ wall suction is observed to decelerate swirl flow whereas injection generates the converse response. As with the tangential flow, backflow (flow reversal) does not arise for even high suction values. With increasing $D a_{\theta}$, temperature values $(H)$ values (figure 13) are markedly elevated throughout the boundary layer, whereas concentration values (I) are reduced. The temperature wall values are in particular strongly increased. Increasing permeability decreases the concentration of solid particles in the regime i.e. increases the presence of voids. This serves to provide greater fluid for the species to diffuse in and elevates thermal diffusion, whereas it counteracts species diffusion. Thermal boundary layer thickness is therefore enhanced whereas concentration boundary layer thickness is reduced, with greater tangential Darcy numbers.

Figs 14-15 depict the effects of tangential Darcy number, $D a_{x}$ i.e. $x$ - direction permeability function, on the velocity distributions, temperature and concentration characteristics. A dramatic acceleration in the tangential velocity is sustained with increasing tangential Darcy number (figure 14); this effect is generally consistent at all values of transverse coordinate. In the tangential momentum equation (18) the $D a_{x}$ parameter arises in the Darcian retarding force term, $-F^{\prime} / D a_{x}$, in a fashion analogous to the presence of $D a_{\theta}$ in the drag force term, $-\frac{G}{D a_{\theta}}$ in eqn. (19). Anisotropy is achieved in the porous medium when $D a_{x} \neq D a_{\theta}$. This provides an alternate approach to that propounded by other researchers e.g. Kumar and Bera [32] wherein an anisotropic permeability ratio is employed, preventing individual variation of hydraulic conductivity properties in different directions in terms of a discrete Darcy number. Increasing $D a_{x}$ evidently reduces the tangential Darcian drag force which will effectively accelerate 
tangential flow. Conversely a weak deceleration in swirl flow is computed with large increase in $D a_{x}$, since the gain in tangential momentum is compensated for by a fall in swirl momentum. Both temperature $(H)$ and concentration $(I)$, are significantly reduced (figure 15) with greater swirl Darcy number, $D a_{x}$. As indicated earlier with progressive increase in permeability, the regime comprises a lower quantity of solid material fibers; this provides a greater volume of fluid for tangential momentum to be elevated and swirl momentum to be stifled. The latter results in suppression of both thermal and species diffusion with greater swirl Darcy number values which manifests in a thinning in both the thermal and species boundary layer thicknesses. Concentration magnitudes are however greater again than temperatures.

\section{CONCLUSIONS}

A theoretical and numerical study of radiative non-isothermal, non-isosolutal (coupled heat and mass transfer) steady laminar boundary layer natural convection flow from a rotating permeable vertical cone to anisotropic Darcian porous medium with multiple surface slip effects, has been conducted. The porous medium has high permeability. The governing equations have been rendered into self-similar form with appropriate scaling transformations, subject to physically realistic boundary conditions incorporating linear temperature and concentration distributions at the cone surface and momentum, thermal and mass (solutal) slip effects. MAPLE quadrature numerical solutions are developed for the resulting ordinary differential boundary value problem. Validation has been achieved with previous non-porous solutions of Ece (2006) and also with Keller's box method achieving close agreement. The present study has shown that greater thermal radiation flux enhances temperatures whereas it reduces concentration magnitudes. With greater momentum slip tangential flow and temperatures are enhanced, whereas swirl flow and concentration magnitudes are reduced. Increasing thermal slip however damps the tangential flow and reduces temperatures whereas it elevates concentrations and accelerates the swirl flow. Increasing solutal slip effectively decreases concentration.

Tangential and swirl velocities are observed to be generally elevated with increasing permeability functions (i.e. $\theta$ and $x$-direction Darcy parameters). The present investigation considers Newtonian fluids. Future studies will address a variety of rheological liquids and will be communicated imminently. 


\section{References}

Alex, S.M. and Patil, P.R., Effect of a variable gravity field on convection in an anisotropic porous medium with internal heat source and inclined temperature gradient, ASMEJ. Heat Transfer, vol. 124, no. 1, pp. 144-150,2001.

Anachalam, G., Chamkha, A.J., Sundarammal, K. and Mohanakrishnan, V., Chemical reaction effects on unsteady MHD free convective flow in a rotating porous medium with mass transfer, Thermal Science, Vol. 18, pp. 515-526, 2014.

Al-Harbi, S., Self-similar solution of heat and mass transfer of unsteady mixed convection flow on a rotating cone embedded in a porous medium saturated with a rotating fluid, Appl. Math., vol. 2, pp. 1196-1203,2011.

Bég, O.A., Takhar, H.S., Zueco, J., Sajid, A. and Bhargava, R., Transient Couette flow in a rotating non-Darcian porous medium parallel plate configuration: network simulation method solutions, Acta Mechanica, vol. 200, pp. 129-144,2008.

Bég, O.A., Khan, W.A., and Uddin, M.J., Multiple slip effects on unsteady magnetohydrodynamic nanofluid transport with heat generation/absorption effects in temperature dependent porous media, J. Porous Med., vol. 18, no. 9, 2015.

Bég, O.A., and Makinde, O.D., Viscoelastic flow and species transfer in a Darcian highpermeability channel, Petroleum Sci. and Eng., vol. 76, pp. 93-99, 2011.

Bég, O.A., Numerical methods for multi-physical magnetohydrodynamics, Chapter 1, pp. 1-112, New Developments in Hydrodynamics Research, Nova Science, New York, September, 2012.

O. Anwar Bég, O.D. Makinde, J. Zueco and S.K. Ghosh, Hydromagnetic viscous flow in a rotating annular high-porosity medium with nonlinear Forchheimer drag effects: numerical study, World J. Model. and Simul., 8, 83-95 (2012).

Bég, O.A., Uddin, M.J., Rashidi, M.M., and Kavyani, N., Double-diffusive radiative magnetic mixed convective slip flow with Biot and Richardson number effects, J. Eng. Thermophy., vol. 23, no. 2, pp. 79-97, 2014.

Blums, E, Mikhailov, Yu, and Ozols, R. Heat and Mass Transfer in MHD Flows, World Scientific, 1987, Singapore.

Bogatyreva, N., Bartlova, M., Aubrecht, V. and Holcman, V., P1-approximation for radiative transfer: application to $\mathrm{SF}_{6}+\mathrm{Cu}$ arc plasmas, Open Chem., vol. 13, pp. 502$508,2015$.

Cherif, B. and Sifaoui, M.S., Theoretical study of heat transfer by radiation, conduction and convection in a semi-transparent porous medium in a cylindrical enclosure, $J$. Quantitative Spectroscopy and Radiative Heat Transfer, vol. 83, pp. 519-527, 2004.

Cogley, A.C.L., Vincenti, W.G. and Gilles, E.S., Differential approximation for radiative heat transfer in a nonlinear equations-grey gas near equilibrium, AIAA J., vol. 6, pp. 551 - 553, 1968. 
Dembele, S., Wen, J.X., and Sacadura, J.F., Analysis of the two-flux model for predicting water spray transmittance in fire protection application, ASME J. Heat Transfer, vol. 122, pp. 183-186, 2000.

Dhanasekaran, M.R., Das, S.K. and Venkateshan, S.P., Natural convection in a cylindrical enclosure filled with heat generating anisotropic porous medium, ASME J. Heat Transfer, vol. 124, no. 1, pp. 203-207, 2001.

Dehghan, A.A. and Behnia, M., Combined natural convection - conduction and radiation heat transfer in a discretely heated open cavity, ASME J. Heat Transfer, vol. 118, pp. 5664,1996 .

Dahan, N., Jehl, Z., Guillemoles et al., Using radiative transfer equation to model absorption by thin $\mathrm{Cu}(\mathrm{In}, \mathrm{Ga}) \mathrm{Se} 2$ solar cells with Lambertian back reflector, Opt Express. Vol. 21, no. 3, pp.: 2563-80,2013.

Ece, M.C., Free convection flow about a vertical spinning cone under a magnetic field, Appl. Math. and Compu., vol.179, pp. 231-242, 2006.

Fang, T., Flow and mass transfer for an unsteady stagnation-point flow over a moving wall considering blowing effects, ASME J. Fluids Eng., vol. 136, no. 7, pp. 071103, 2014.

Fang, T. and Jing, W., Flow, heat, and species transfer over a stretching plate considering coupled Stefan blowing effects from species transfer, Commun. in Nonlin. Sci. and Numer. Simulat., vol. 19, no. 9, pp. 3086-3097, 2014.

Frenot, A. and Chronakis, I.S., Polymer nanofibers assembled by electrospinning, Current Opinion in Colloid and Interface Science, vol. 8, pp. 64-75, 2003.

Holeschovsky, U.B. and Cooney, C.L., Quantitative description of ultra-filtration in a rotating filter device, AIChemE J., vol. 37, pp. 1219-1226, 1991.

Javed, T., Sajid, M., Abbas, Z. and Ali, N., Non-similar solution for rotating flow over an exponentially stretching, Int. J. Numer. Meth. for Heat and Fluid Flow, vol. 21, no., 7, pp. 903-908,2011.

Javed, T., Abbas, Z., Hayat, T. and Asghar, S., Homotopy analysis for stagnation slip flow and heat transfer on a moving plate, ASME J. Heat Transfer, vol. 131, no.9, pp. 094506, 2009.

Kamdem, H.T.T. and Baillis, D.D., Reduced models for radiative heat transfer analysis through anisotropic fibrous medium, ASME J. Heat Transfer, vol. 132, no. 7, pp. 072703 2010 .

Keller, H.B., Numerical Solution of Two-Point Boundary Value Problems, SIAM, Philadelphia, USA, 1976.

Kumar, P., and Frederic T.., Impact of anisotropy on geometrical and thermal conductivity of metallic foam structures, J. Porous Med., vo. 18, no. 10, 2015. 
A.Kumar and P. Bera, Natural convection in an anisotropic porous enclosure due to nonuniform heating from the bottom wall, ASME J. Heat Transfer 131(7), 072601 (2009).

A.A. Kendoush, Similarity solution for heat convection from a porous rotating disk in a flow field, ASME J. Heat Transfer 135(8), 084505 (2013).

J. Lahjomri and A. Oubarra, Hydrodynamic and thermal characteristics of laminar slip flow over a horizontal isothermal flat plate, ASME J. Heat Transfer 135(2), 021704 (2013

Lienhard IV JH and Lienhard VJH. A Heat Transfer Textbook. 3rd ed. Cambridge, MA: Phlogiston Press; 2005, 662-663.

I. Malico, Numerical study on the influence of radiative properties in porous media combustion, ASME J. Heat Transfer, 123, 951-957 (2001).

M.F. Modest, Radiative Heat Transfer, MacGraw-Hill, USA (1993).

F. Moufekkir, M.A. Moussaoui, A. Mezrhab, J.P. Fontaine and M. Bouzidi, Investigation of double diffusive natural convection in presence of gray gas radiation within a square cavity using multiple relaxation time Lattice Boltzmann method, ASME J. Heat Transfer, 135(10):102701-102701-10 (2013).

Nellis G and Klein S. Heat Transfer. Cambridge University Press; 2008 [Chapter 9, p. E23-5].

A. Oubarra, A. Sadeghi, M. Baghani and M. H. Saidi, Gaseous slip flow mixed convection in vertical microducts with constant axial energy input, ASME J. Heat Transfer 136(3), 032501 (2013).

V.R. Prasad, B. Vasu, O. Anwar Bég and D.R. Parshad, Thermal radiation effects on magnetohydrodynamic free convection heat and mass transfer from a sphere in a variable porosity regime, Commun. in Nonl. Sci. and Numer. Simul., 17, 654-671 (2012).

Saxena and A.C. Srivastava, Rotation of a solid sphere in a viscous fluid bounded by a concentric spherical porous medium, J. Porous Media, 11, 575-588 (2008).

Shiping Y. and T.A. Ameel, Slip flow convection in isoflux rectangular microchannels, ASME J. Heat Transfer 124(2), 346-355 (2001).

H.S. Takhar, O. Anwar Bég and M. Kumari, Computational analysis of coupled radiation convection dissipative flow in a porous medium using the Keller-Box implicit difference scheme, Int. J. Energy Research, 22,141-159 (1998).

C. Tierney, S. Wood, A.T. Harris and D.F. Fletcher, Computational fluid dynamics modelling of porous burners, $7^{\text {th }}$ Int Conf. CFD in Minerals and Process Industries, CSIRO, Melbourne, Australia, Dec. 9-11 (2009).

M.J. Uddin, O. Anwar Bég and A.I.M. Ismail, Mathematical modelling of radiative hydromagnetic thermo-solutal nanofluid convection slip flow in saturated porous media, 
Math. Prob. Engineering. 2014, Article ID 179172,

M.J. Uddin, O. Anwar Bég and N.S. Amin, Hydromagnetic transport phenomena from a stretching or shrinking nonlinear nanomaterial sheet with Navier slip and convective heating: a model for bio-nano-materials processing, J. Magnetism Magnetic Materials, 368, 252-261(2014).

M.J. Uddin, N H M. Yusoff, O Anwar Bég and A.I.M. Ismail, Lie group analysis and numerical solutions for non-Newtonian nanofluid flow in a porous medium with internal heat generation, Phy. Scr., 87, 1-14 (2013).

M.J. Uddin, W.A. Khan and A.I.M. Ismail, Free convection boundary layer flow from a heated upward facing horizontal flat plate embedded in a porous medium filled by a nanofluid with convective boundary condition, Transp. Porous Med., 92, 867-881 (2012).

M.J. Uddin, O. Anwar Bég and A.I.M. Ismail, Mathematical modelling of radiative hydromagnetic thermo-solutal nanofluid convection slip flow in saturated porous media, Math. Prob. Eng., 2014, Article ID 179172, 11 pages: doi.org/10.1155/2014/179172 (2014)

R. Viskanta, Radiation heat transfer in materials processing and manufacturing, Energy and the Environment, 15, 101-112 (1999).

S.R. Varnas and J.S. Truelove, Simulating radiative transfer in flash smelting furnaces, Applied Mathematical Modelling, 19, 456-464 (1995).

Wijewardane, S. and Goswami, D.Y., A review on surface control of thermal radiation by paints and coatings for new energy applications, Renew. Sust. Energy Revs., vol.16, 1863-1873 (2012).

Yang, G. and Ebadian, M.A., Thermal radiation and laminar forced convection in the entrance of a pipe with axial conduction and radiation, Int. J. Heat Fluid Flow, vol. 12, , pp.202-209, 1991. 


\section{Figures Captions}

Figure 1: Physical model and coordinate system

Fig. 2 Effect of wall transpiration $(S)$ on tangential velocity $\left(F^{\prime}\right)$ and swirl velocity $(G)$.

Fig.3 Effect of wall transpiration $(S)$ on temperature $(H)$ and concentration $(I)$.

Fig.4 Effect of velocity slip $(a)$ on tangential velocity $\left(F^{\prime}\right)$ and swirl velocity $(G)$.

Fig.5 Effect of velocity slip $(a)$ on the temperature $(H)$ and concentration $(I)$.

Fig.6 Effect of thermal slip $(b)$ on the tangential velocity $\left(F^{\prime}\right)$ and swirl velocity $(G)$.

Fig.7 Effect of thermal slip $(b)$ on temperature $(H)$ and concentration $(I)$.

Fig.8 Effect of mass slip (c) on concentration $(I)$.

Fig. 9 Effect of conduction-radiation $\left(R_{c}\right)$ on temperature $(H)$ and concentration $(I)$.

Fig.10 Effect of buoyancy ratio $(N)$ on tangential velocity $\left(F^{\prime}\right)$ and swirl velocity $(G)$.

Fig.11 Effect of buoyancy ratio $(N)$ on temperature $(H)$ and concentration $(I)$.

Fig.12 Effect of swirl Darcy number $\left(D a_{\theta}\right)$ on tangential $\left(F^{\prime}\right)$ and swirl velocity $(G)$.

Fig.13 Effect of swirl Darcy number $\left(D a_{\theta}\right)$ on temperature $(H)$ and concentration $(I)$.

Fig.14 Effect of tangential Darcy number $\left(D a_{x}\right)$ on tangential $\left(F^{\prime}\right)$ and swirl velocity $(G)$.

Fig.15 Effect of tangential Darcy number $\left(D a_{x}\right)$ on temperature $(H)$ and concentration (I). 